\title{
Medical Image of the Week: Boerhaave's Syndrome During Colonoscopy
}

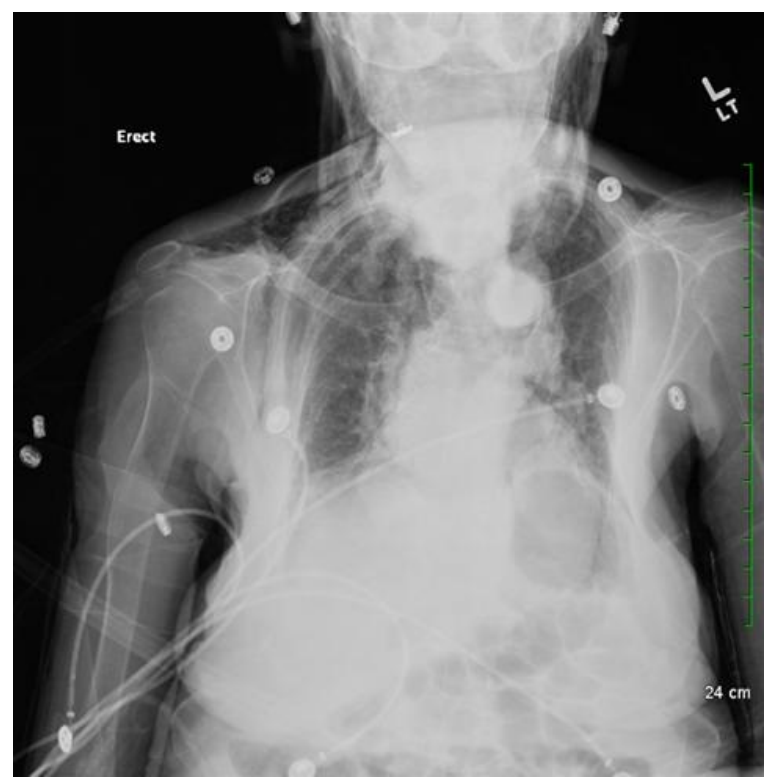

Figure 1. Chest $X$ ray showing bilateral subcutaneous emphysema extending from the supraclavicular area and above to the neck.

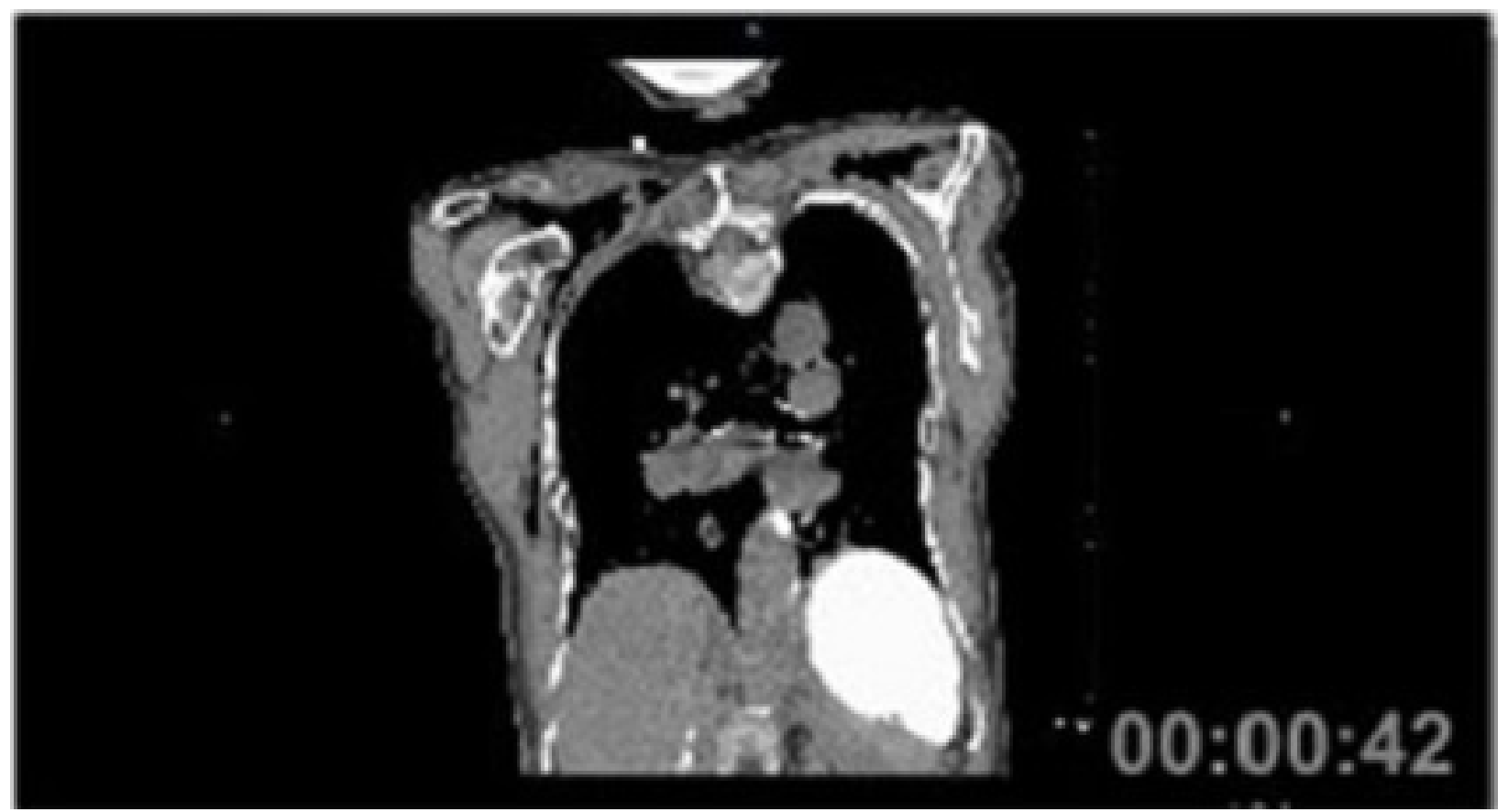

Figure 2. Representative coronal views of the thoracic CT scan showing subcutaneous emphysema in the supraclavicular area and neck. 


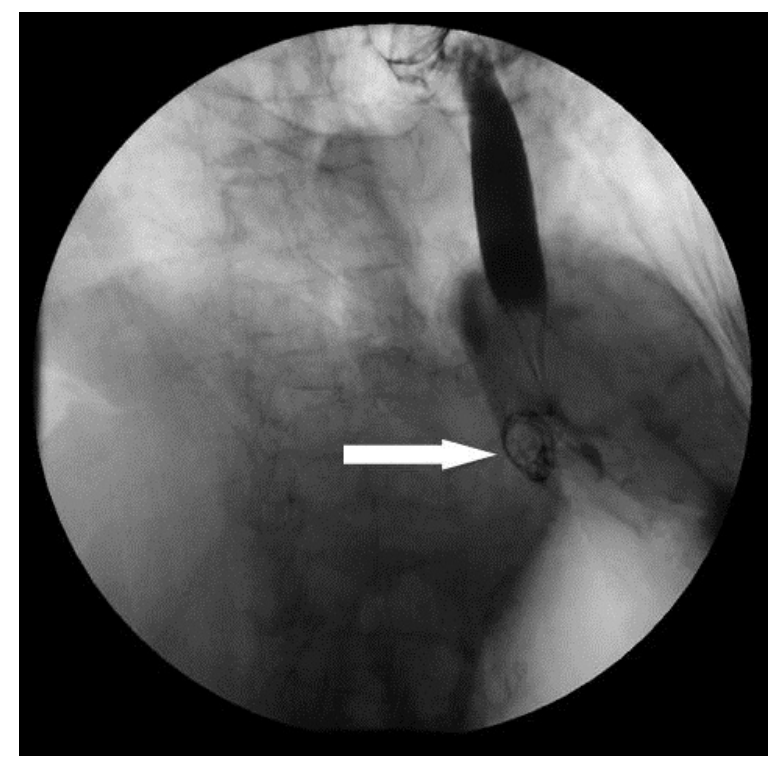

Figure 3. Fluoroscopic esophagram revealing a focus of oral contrast actively extravasating (white arrow) approximately at $2.5 \mathrm{~cm}$ above the gastro-esophageal junction consistent with a small perforation.

A 76-year-old woman with no significant past medical history underwent outpatient screening colonoscopy. The procedure was difficult due to a tortuous colon and only multiple diverticula were visualized. She vomited once during the procedure. In the immediate postoperative period, she complained of neck swelling. Her vital signs were stable. On examination, right sided neck and facial swelling with palpable crepitations were noticed as well as coarse breath sounds heard on auscultation of both lung fields. Immediate chest X-ray (Figure 1) was obtained which showed bilateral subcutaneous emphysema extending from the supraclavicular area and above to the neck. Subsequent thoracic CT scan showed extensive subcutaneous air within the soft tissues of the neck bilaterally, extending into the mediastinum and along the anterior chest wall (Figure 2). An esophagram (Figure 3 ) revealed a focus of oral contrast actively extravasating approximately at $2.5 \mathrm{~cm}$ above the gastro-esophageal junction consistent with a small perforation. She underwent left thoracotomy with esophageal repair. Further hospital course was uncomplicated and she was discharged to a subacute rehabilitation facility.

Boerhaave's syndrome is a spontaneous perforation of the esophagus due to sudden increase in intra-esophageal pressure combined with negative intrathoracic pressure caused by straining or vomiting (1). The tear usually occurs at the left posterolateral wall of the lower third of the esophagus. Usually patients have severe retching and vomiting 
which is followed by excruciating retrosternal chest and upper abdominal pain after perforation. Other manifestations are odynophagia, tachypnea, dyspnea, fever, and shock. On physical examination subcutaneous emphysema (crepitation) is an important diagnostic feature. Chest radiograph usually reveals mediastinal or free peritoneal air as the initial manifestation, and hours to days later pleural effusion with or without pneumothorax, widened mediastinum, and subcutaneous emphysema are typically seen. The diagnosis of esophageal perforation can also be confirmed by water-soluble contrast esophagram using Gastrograffin, which reveals the location and extent of extravasation of contrast. Treatment depends upon the size and location of the perforation. Surgery is generally required for thoracic perforations while cervical perforations can often be managed conservatively with continuous nasogastric suction, intravenous broad-spectrum antibiotics, and parenteral nutrition.

Chandramohan Meenakshisundaram MD, Nanditha Malakkla MD and Venu Ganipisetti MD

Department of Internal Medicine

Presence Saint Francis Hospital

Evanston, IL USA

\section{Reference}

1. Nirula R. Esophageal perforation. Surg Clin North Am. 2014;94(1):35-41. [CrossRef] [PubMed] 